\title{
Sufficient conditions for thermal rectification in hybrid quantum structures
}

\author{
Lian-Ao $\mathrm{Wu}^{1,2}$ and Dvira Segal ${ }^{1}$ \\ ${ }^{1}$ Chemical Physics Group, Department of Chemistry and Center for Quantum Information and Quantum Control, \\ University of Toronto, 80 St. George street, Toronto, Ontario, M5S 3H6, Canada and \\ ${ }^{2}$ Department of Theoretical Physics and History of Science, \\ The Basque Country University (EHU/UPV), PO Box 644, 48080 Bilbao, Spain
}

(Dated: November 4, 2018)

\begin{abstract}
We analytically identify sufficient conditions for manifesting thermal rectification in two-terminal hybrid structures within the quantum master equation formalism. We recognize two classes of rectifiers. In type A rectifiers the contacts are dissimilar. In type B rectifiers the contacts are equivalent, but the system and baths have different particle statistics, and the system is (parametrically) asymmetrically coupled to the baths. Our study applies to various hybrid junctions including metals, dielectrics, and spins.
\end{abstract}

PACS numbers: 63.22.-m, 44.10.+i, 05.60.-k, 66.70.-f

Understanding heat transfer in hybrid structures is of fundamental and practical importance for controlling transport at the nanoscale, and for realizing functional devices [1]. Among the systems that fall into this category are metal-molecule-metal junctions, the basic component of molecular electronic devices [2], and dielectric-molecule-dielectric systems, where vibrational energy flow activates reactivity and controls dynamics [3]. Phononic junctions are also captivating and essential for understanding the validity of the Fourier's law of thermal conduction at the nanoscale [4, 5]. Single-mode radiative heat conduction between ohmic metals was recently detected, showing that photonic thermal conductance is quantized [6]. Other hybrid systems with interesting thermal properties are electronic spin-nuclear spin interfaces [7], metal-molecule contacts with exciton to phonon energy transfer [8], and metal-superconductor junctions [9].

Thermal rectification, namely an asymmetry of the heat current for forward and reversed temperature gradients, has recently attracted considerable theoretical 10, 11, 12, 13, 14, 15, 16] and experimental 17, 18] attention. Most theoretical studies, confined to a specific realization, have analyzed this phenomenon in phononic systems using classical molecular dynamics simulations. In this letter we attempt a first step towards an analytical understanding of this effect. We establish sufficient conditions for manifesting thermal rectification in a prototype-hybrid quantum model, including a central quantized unit (subsystem) and two bulk objects (reservoirs). We identify two classes of thermal rectifiers: (i) Type A rectifiers where the terminals are dissimilar i.e. of different mean energy (or heat capacity). (ii) Type B rectifiers, where the contacts are equivalent, but the reservoirs and subsystem have different statistics, combined with unequal coupling strengths at the two ends. We manifest that these rectifiers could be realized in several subsystems (harmonic and anharmonic) and reservoirs (spin, metal, dielectrics), see Fig. 1.

Consider a 1-dimensional hybrid structure where a central unit $H_{S}$ interacts with two reservoirs $H_{\nu}^{0}(\nu=L, R)$

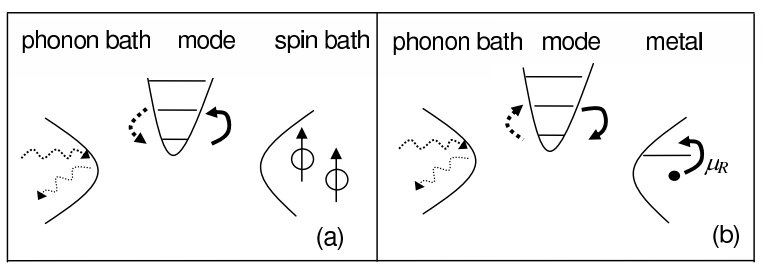

FIG. 1: Examples of two hybrid systems treated in this work. (a) Single mode heat transfer between a solid and a spin bath. (b) Phonon to exciton energy exchange. The central unit can represent either a vibrational or a radiation mode.

of temperatures $T_{\nu}=\beta_{\nu}^{-1}$ via the coupling terms $V_{\nu}$,

$$
H=H_{L}^{0}+H_{R}^{0}+H_{S}+V_{L}+V_{R} .
$$

The heat current from the left bath into the subsystem is given by $J_{L}=\frac{i}{2} \operatorname{Tr}\left(\left[H_{L}^{0}-H_{S}, V_{L}\right] \rho\right) ;(\hbar \equiv 1)[19]$, where $\rho$ is the total density matrix, and we trace over the system and reservoirs degrees of freedom. In steady-state the expectation value of the interaction is zero, $\operatorname{Tr}\left(\frac{\partial V_{L}}{\partial t} \rho\right)=$ 0 , and we obtain

$$
J=i \operatorname{Tr}[\widehat{J} \rho] ; \quad \widehat{J}=\frac{i}{2}\left[V_{L}, H_{S}\right]+\frac{i}{2}\left[H_{S}, V_{R}\right] .
$$

This expression was derived based on the equality $J_{L}=$ $-J_{R}$ in steady-state. We discard the subscript $L$ in (2), thus the heat current is defined positive when flowing left to right. The system Hamiltonian assumes a diagonal form, and we also consider separable couplings

$$
\begin{aligned}
H_{S} & =\sum_{n} E_{n}|n\rangle\langle n| ; \\
V_{\nu} & =S B_{\nu} ; \quad S=\sum_{n, m} S_{m, n}|m\rangle\langle n| .
\end{aligned}
$$

Here $S$ is a subsystem operator and $B_{\nu}$ is an operator in terms of the $\nu$ bath degrees of freedom. For simplicity we set $S_{m, n}=S_{n, m}$. In what follows we consider cases where $B_{L}$ and $B_{R}$ have equal structure but different prefactors. We refer to this case as "parametric asymmetry", 
or "unequal coupling strength", rather than "functional asymmetry", resulting from dissimilar B's. Note that if the commutator $\left[H_{S}, S\right]=0$, the heat current trivially vanishes.

We begin and discuss the type A rectifier, constructed by adopting reservoirs with distinct properties, as we explain below. First we derive a general expression for the heat flux in hybrid structures. Formally, if we write the heat current as $J\left(T_{a}, \Delta\right)=\sum_{k} \alpha_{k}\left(T_{a}\right) \Delta^{k}$, rectification takes place if even terms survive, $\alpha_{k=2 n} \neq 0 ; n=1,2 \ldots$, $T_{a}=T_{L}+T_{R}$ and $\Delta=T_{L}-T_{R}$.

The initial density matrix $\rho$ is assumed to be a tensor product of system $\rho_{S}$ and bath $\rho_{B}=\rho_{L}\left(T_{L}\right) \otimes \rho_{R}\left(T_{R}\right)$ factors, where $\rho_{\nu}(T)=e^{-\frac{H_{\nu}^{0}}{T}} / \operatorname{Tr}_{\nu}\left[e^{-\frac{H_{\nu}^{0}}{T}}\right](\nu=L, R)$. For convenience we delete the direct reference to time. In terms of $T_{a}$ and $\Delta$ we can write

$$
\rho\left(T_{a}, \Delta\right)=\frac{1}{Z} e^{-\frac{2 T_{a}\left(H_{L}^{0}+H_{R}^{0}\right)-2 \Delta\left(H_{L}^{0}-H_{R}^{0}\right)}{T_{a}^{2}-\Delta^{2}}} \otimes \rho_{S},
$$

where $Z=\operatorname{Tr}\left[\rho_{S} \rho_{B}\right]$ is the partition function with the trace performed over bath and system degrees of freedom. Furthermore, without loss of generality, the system density matrix is assumed to depend initially only on the average temperature, $\rho_{S} \equiv \rho_{S}^{(0)}\left(T_{a} / 2\right)$.

The expectation value of the energy current is given by evaluating $J\left(T_{a}, \Delta\right)=\operatorname{Tr}\left[\widehat{J} \rho\left(T_{a}, \Delta\right)\right]$, where $\widehat{J}$ is an Heisenberg representation operator $\widehat{J}=e^{i H t} \widehat{J}(0) e^{-i H t}$. Expanded in powers of $\Delta$ we get

$$
J\left(T_{a}, \Delta\right)=\frac{2 \Delta}{T_{a}^{2}} \alpha_{0}\left(T_{a}\right)+\frac{(2 \Delta)^{2}}{T_{a}^{4}} \alpha_{1}\left(T_{a}\right)+O\left(T_{a}, \Delta^{3}\right),
$$

with the coefficients

$$
\begin{aligned}
\alpha_{0}\left(T_{a}\right) & =\operatorname{Tr}\left[\widehat{J}\left(H_{L}^{0}-H_{R}^{0}\right) \rho_{T}\right], \\
\alpha_{1}\left(T_{a}\right) & =\frac{1}{2} \operatorname{Tr}\left[\widehat{J}\left(H_{L}^{0}-H_{R}^{0}\right)^{2} \rho_{T}\right] \\
& -\alpha_{0}\left(T_{a}\right) \operatorname{Tr}\left[\left(H_{L}^{0}-H_{R}^{0}\right) \rho_{T}\right] .
\end{aligned}
$$

Here $\rho_{T}=\frac{1}{Z_{T_{a}}} \rho_{L}\left(\frac{T_{a}}{2}\right) \rho_{R}\left(\frac{T_{a}}{2}\right) \rho_{S}^{(0)}\left(\frac{T_{a}}{2}\right)$ is the density matrix at the average temperature $\frac{T_{L}+T_{R}}{2}$ with the partition function $Z_{T_{a}}$. Note that the operators are time dependent, given in their Heisenberg representation. Eq. (7) was derived using the fact that $\operatorname{Tr}\left[\widehat{J} \rho_{T}\right]$ and all its $T$ derivatives are zero. Using the definition of the current operator (2) we obtain an explicit expression for $\alpha_{0}$,

$$
\alpha_{0}\left(T_{a}\right)=i \operatorname{Tr}\left\{\left[S, H_{S}\right]\left(B_{L} H_{L}^{0}+B_{R} H_{R}^{0}\right) \rho_{T}\right\} .
$$

Since $\left[H_{S}, S\right] \neq 0$ [see discussion after Eq. (3)], the linear term in $J$ is finite [20].

We now examine the onset of thermal rectification, i.e. discuss the sufficient conditions for having $\alpha_{1} \neq 0$. While there might be some special values of $T_{a}$ where the two terms in (7) cancel, in general since $B_{\nu}$ and $H_{\nu}^{0}$ are independent operators, the result is finite. Thus, in order to manifest rectification it is enough to analyze when one of the terms in (77) is nonzero. (i) The first expression is finite if $\operatorname{Tr}\left[F H_{L}^{0} \rho_{T}\right] \neq \operatorname{Tr}\left[F H_{R}^{0} \rho_{T}\right]$; $F=\left[S, H_{S}\right]\left(B_{L} H_{L}^{0}+B_{R} H_{R}^{0}\right)$. The second term is nonzero if $\operatorname{Tr}\left[H_{L}^{0} \rho_{T}\right] \neq \operatorname{Tr}\left[H_{R}^{0} \rho_{T}\right]$. Based on (ii) we conclude that rectification emerges when the reservoirs have different mean energy,

$$
\left\langle H_{L}^{0}\right\rangle \equiv \operatorname{Tr}_{L}\left[\rho_{L}(T) H_{L}^{0}\right] \neq \operatorname{Tr}_{R}\left[\rho_{R}(T) H_{R}^{0}\right] \equiv\left\langle H_{R}^{0}\right\rangle .
$$

As an example, consider a bath of 1-dimensional oscillators, $H_{L}^{0}=H_{L}^{k i n}+H_{L}^{\text {pot }}$, where the kinetic energy $H_{L}^{k i n}$ is quadratic in momentum and the potential energy per particle is $C_{n} q^{n} ; n \geq 2$. In the classical limit using the equipartition relation we obtain $\left\langle H_{L}^{0}\right\rangle=T_{L}\left(\frac{1}{2}+\frac{1}{n}\right)$. Thermal rectification thus emerges if the reservoirs have a non-identical power $n$. Note that the separation to three segments $(L$, subsystem, $R$ ) is often artificial, as the system can be practically made of a single structure with a varying potential energy, e.g. an asymmetrically mass loaded nanotube [17]. Our results manifest that such an inhomogeneous structure should rectify heat. Finally, we comment that our discussion could be generalized to cases where $V_{\nu}=S_{\nu} B_{\nu} ; S_{L} \neq S_{R}$, see Eq. (3).

We turn to the type $\mathrm{B}$ rectifier, and show that for equivalent reservoirs rectification emerges when the subsystem and reservoirs have different statistics, in conjunction with some parametric asymmetry. It is easy to show that under (3) the steady-state current (2) becomes $J=$ $i \sum_{n, m} E_{m, n} S_{m, n} \operatorname{Tr}_{\mathrm{B}}\left(B_{L} \rho_{m, n}\right)$, where $E_{m, n}=E_{m}-E_{n}$, and $\operatorname{Tr}_{\mathrm{B}}$ denotes the trace over the $L$ and $R$ reservoirs degrees of freedom. Employing the Liouville equation in the interaction picture, the elements of the total density matrix satisfy

$$
\frac{d \rho_{m, n}}{d t}=-\int_{0}^{t} d \tau[V(t),[V(\tau), \rho(\tau)]]_{m, n}
$$

where $V=V_{L}+V_{R}$, and $V(t)$ are interaction picture operators. Following the standard weak coupling scheme [21], going to the markovian limit, the heat current reduces to

$$
J=\frac{1}{2} \sum_{n, m} E_{m, n}\left|S_{m, n}\right|^{2} P_{n} \times\left(k_{n \rightarrow m}^{L}-k_{n \rightarrow m}^{R}\right),
$$

where the transition rates are given by $k_{n \rightarrow m}^{\nu}=$ $\int_{-\infty}^{\infty} d \tau e^{i E_{n, m} \tau}\left\langle B_{\nu}(\tau) B_{\nu}(0)\right\rangle$, and the population $P_{n}=$ $\operatorname{Tr}_{\mathrm{B}}\left(\rho_{n, n}\right)$ satisfies the differential equation

$$
\dot{P}_{n}=\sum_{\nu, m}\left|S_{m, n}\right|^{2} P_{m} k_{m \rightarrow n}^{\nu}-P_{n} \sum_{\nu, m}\left|S_{m, n}\right|^{2} k_{n \rightarrow m}^{\nu} .
$$

In steady-state $\dot{P}_{n}=0$, and we normalize the population to unity $\sum_{n} P_{n}=1$. Our description to this point is general, as we have not yet specified neither the subsystem nor the interfaces. We consider next two representative models for the system Hamiltonian and its interaction with the baths. In the first model the subsystem is a harmonic oscillator (HO) of frequency $\omega$, 
$H_{S}=\sum_{n} n \omega|n\rangle\langle n|$. This can describe either a local radiation mode [6, 22] or a vibrational mode of the trapped molecule [23]. We also take $S=\sum_{n} \sqrt{n}|n\rangle\langle n-1|+$ c.c, motivated by the bilinear form $V_{\nu} \propto x B_{\nu}, x$ is a subsystem coordinate [23]. This implies that only transitions between nearest states are allowed,

$$
\begin{aligned}
& k^{\nu} \equiv k_{n \rightarrow n-1}^{\nu}=\int_{-\infty}^{\infty} d \tau e^{i \omega \tau}\left\langle B_{\nu}(\tau) B_{\nu}(0)\right\rangle ; \\
& k_{n-1 \rightarrow n}^{\nu}=e^{-\beta_{\nu} \omega} k_{n \rightarrow n-1}^{\nu} .
\end{aligned}
$$

Solving (12) in steady-state using the rates (13), the heat current (11) can be analytically calculated,

$$
J^{(H O)}=-\frac{\omega\left[n_{B}^{L}(\omega)-n_{B}^{R}(\omega)\right]}{n_{B}^{L}(-\omega) / k^{L}+n_{B}^{R}(-\omega) / k^{R}},
$$

where $n_{B}^{\nu}(\omega)=\left[e^{\beta_{\nu} \omega}-1\right]^{-1}$ is the Bose-Einstein distribution function at $T_{\nu}=1 / \beta_{\nu}$.

Our second subsystem is a two-level system (TLS). Here $H_{S}=\frac{\omega}{2} \sigma_{z}$, and we employ a nondiagonal interaction $S=\sigma_{x}$. These terms can represent an electronic spin rotated by the environment [7]. It can also describe an anharmonic (truncated) molecular vibration dominating heat flow through the junction [12, 23]. Re-calculating the long-time population (12), the heat flux reduces to

$$
J^{(T L S)}=\frac{\omega\left[n_{S}^{L}(\omega)-n_{S}^{R}(\omega)\right]}{n_{S}^{L}(-\omega) / k^{L}+n_{S}^{R}(-\omega) / k^{R}}
$$

with the rates (13) and the spin occupation factor $n_{S}^{\nu}(\omega)=\left[e^{\beta_{\nu} \omega}+1\right]^{-1}$. Expressions (14) and (15) show that in the weak coupling limit the effect of the environment enters only through the relaxation rates $k^{\nu}$, evaluated at the subsystem energy spacing $\omega$.

We now analyze the general structure of the last two expressions, and discuss the onset of thermal rectification. It is clear that if $k^{L}(T)=k^{R}(T)$, i.e. the reservoirs and system-bath interactions $B_{\nu}$ are equivalent, thermal rectification is absent as $J(\Delta)=-J(-\Delta)$. On the other hand, if $k^{L}(T)=f(T) k^{R}(T)$, resulting e.g. from the use of dissimilar reservoirs, the system generally rectifies heat besides some special points in the parameter space, depending on the details of the model. This case reduces to the type A rectifier discussed above.

However, a more careful analysis of Eqs. (14) and (15) reveals that rectification prevails if $k^{L}(T)=c k^{R}(T)$, $c \neq 1$ is a constant, given that the relaxation rates' temperature dependence differs from the central unit particle statistics. For example, for a spin (TLS) subsystem we require that $n_{S}^{\nu}(-\omega) / k^{\nu}=g\left(T_{\nu}\right)$, a non-constant function of temperature. A TLS asymmetrically coupled to two harmonic baths thus rectifies heat 12. As we show next, the temperature dependence of the rates $k^{\nu}$ reflects the reservoirs statistics. We therefore classify type $\mathrm{B}$ rectifiers as junctions where the system and bath differ in their statistics, and the equivalent reservoirs are (parametrically) asymmetrically coupled to the system. We specify next the contacts, and exemplify the two classes of thermal rectifiers in various hybrid structures.

Spin bath. Assuming the environment includes a set of distinguishable noninteracting spin- $1 / 2$ particles $(p=1,2, . ., P)$, the bath Hamiltonian is given by summing all separable contributions, $H_{\nu}^{0}=\sum_{p} h_{\nu, p}^{0}$ and $B_{\nu}=\sum_{p} b_{\nu, p}$. The relaxation rate (13) reduces to

$$
k^{\nu}=n_{S}^{\nu}(-\omega) \Upsilon_{\nu}(\omega)
$$

where $n_{S}^{\nu}(\omega)=\left[e^{\beta_{\nu} \omega}+1\right]^{-1} \quad$ and $\quad \Upsilon_{\nu}(\omega) \quad=$ $2 \pi \sum_{p}\left|\left\langle\left. 0\right|_{p} b_{\nu, p} \mid 1\right\rangle_{p}\right|^{2} \delta\left(\omega+\epsilon_{p}(0)-\epsilon_{p}(1)\right)$, with the $p$-particle eigenstates $|i\rangle_{p}$ and eigenvalues $\epsilon_{p}(i)(i=0,1)$.

Solid/Radiation field (harmonic bath). This bath includes a set of independent harmonic oscillators, creation operator $a_{\nu, j}^{\dagger}$. System-bath interaction is further assumed to be bilinear, $H_{\nu}^{0}=\sum_{j} \omega_{j} a_{\nu, j}^{\dagger} a_{\nu, j} ; B_{\nu}=$ $\sum_{j} \lambda_{\nu, j}\left(a_{\nu, j}+a_{\nu, j}^{\dagger}\right)$, where $\lambda_{\nu, j}$ are the system-bath coupling elements. This leads to the relaxation rate (13)

$$
k^{\nu}=-\Gamma_{B}^{\nu}(\omega) n_{B}^{\nu}(-\omega),
$$

where $\Gamma_{B}^{\nu}(\omega)=2 \pi \sum_{j}\left|\lambda_{\nu, j}\right|^{2} \delta\left(\omega_{j}-\omega\right)$ is an effective system-bath coupling energy and $n_{B}^{\nu}$ is the Bose-Einstein distribution function at temperature $T_{\nu}$.

Metal. As a final example the contact is made metallic, including a set of noninteracting spinless electrons, creation operator $c_{\nu, i}^{\dagger}$, The bath operator coupled to the system allows scattering between electronic states within the same lead, $H_{\nu}^{0}=\sum_{i} \epsilon_{i} c_{\nu, i}^{\dagger} c_{\nu, i} ; B_{\nu}=\sum_{i, j} v_{\nu, i, j} c_{\nu, i}^{\dagger} c_{\nu, j}$. The transition rate (13) can be written as

$$
\begin{aligned}
k^{\nu}= & -2 \pi n_{B}^{\nu}(-\omega) \sum_{i, j}\left|v_{\nu, i, j}\right|^{2} \delta\left(\epsilon_{i}-\epsilon_{j}+\omega\right) \\
& \times\left[n_{F}^{\nu}\left(\epsilon_{i}\right)-n_{F}^{\nu}\left(\epsilon_{i}+\omega\right)\right],
\end{aligned}
$$

with the Fermi-Dirac distribution function $n_{F}^{\nu}(\epsilon)=$ $\left[e^{\beta_{\nu}\left(\epsilon-\mu_{\nu}\right)}+1\right]^{-1}$ at the chemical potential $\mu_{\nu}$. One could also write

$$
k^{\nu}=-n_{B}^{\nu}(-\omega) \Lambda^{\nu}\left(T_{\nu}, \omega\right),
$$

where $\Lambda^{\nu}\left(T_{\nu}, \omega\right)=2 \pi \int d \epsilon\left[n_{F}^{\nu}(\epsilon)-n_{F}^{\nu}(\epsilon+\omega)\right] F_{\nu}(\epsilon)$. The function $F_{\nu}(\epsilon)=\sum\left|v_{\nu, i, j}\right|^{2} \delta\left(\epsilon-\epsilon_{j}+\omega\right) \delta\left(\epsilon_{i}-\epsilon\right)$ depends on the system-bath coupling elements and the specific band structure. Assuming that the density of states slowly varies in the energy window $\omega$, this function could be expanded around the chemical potential [22]. If the Fermi energy is much bigger than the conduction band edge we obtain $\Lambda^{\nu}\left(T_{\nu}, \omega\right) \approx \Gamma_{F}^{\nu}\left(1+\delta_{\nu} \frac{T_{\nu}}{\mu_{\nu}}\right)$, where $\Gamma_{F}^{\nu}=2 \pi \omega F_{\nu}\left(\mu_{\nu}\right)$, and $\delta$ is a constant of order one, measuring the deviation from a flat band structure near the chemical potential [22].

Consider for example a type A solid-HO-metal rectifier, representing an electronic to vibrational energy conversion device, see Fig. 1(b). This system may be realized by attaching an insulating molecule to a metal (STM 


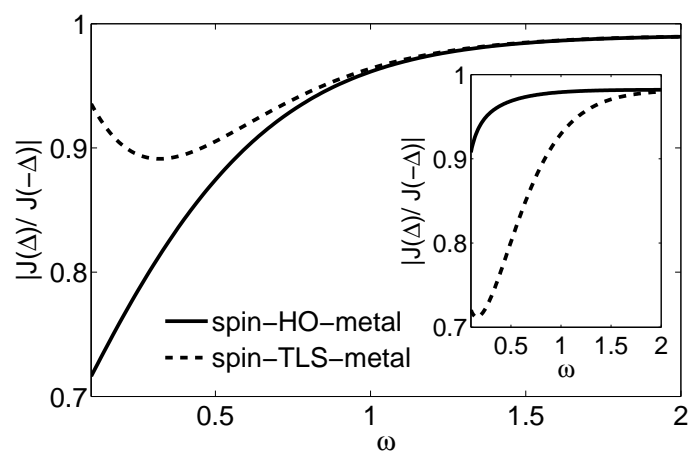

FIG. 2: Spin-HO-Metal rectifier (full) and a Spin-TLS-Metal rectifier (dashed). Rectification ratio is presented as a function of the subsystem spacing. $T_{a}=0.5, \Delta=0.1, \delta_{R}=0.2$, $\mu_{R}=1$, Main plot: The subsystem is equally coupled to the two ends, $\Upsilon_{L}=\Gamma_{F}^{R}=1$. Inset: The effect can be tuned by manipulating system-bath couplings, $\Upsilon_{L}=1, \Gamma_{F}^{R}=0.05$.

tip), while the underneath surface is insulating. Setting the coupling strength at both contacts to be the same, $\Gamma_{B}^{L}(\omega)=\Gamma_{F}^{R}(\omega)$, we can calculate the rectification ratio $\mathcal{R} \equiv\left|\frac{J(\Delta)}{J(-\Delta)}\right|$ using (14), (17) and (19)

$$
\mathcal{R}=\frac{\left(2+\delta_{R} \frac{T_{L}}{\mu_{R}}\right)\left(1+\delta_{R} \frac{T_{R}}{\mu_{R}}\right)}{\left(1+\delta_{R} \frac{T_{L}}{\mu_{R}}\right)\left(2+\delta_{R} \frac{T_{R}}{\mu_{R}}\right)} \sim 1-\Delta \frac{\delta_{R}}{2 \mu_{R}} .
$$

Therefore, if the metal density of state varies with energy $(\delta \neq 0)$, thermal rectification is presented $[22$. Interestingly, we can show that in a solid-TLS-metal junction $\mathcal{R}$ could be modulated to be greater or smaller than one by varying the gap $\omega$. Thus, phonon-to-exciton heat conversion can be made effective, while the exciton-to-phonon route becomes ineffective, and vice versa. As a secondtype B- example consider a solid-HO-solid structure. In the classical limit [see (15) and (17)] we get

$$
\begin{aligned}
J\left(T_{a}, \Delta\right) & =\frac{\omega \Gamma_{B}^{L} \Gamma_{B}^{R} \Delta}{2 \Gamma_{B}^{L} T_{L}+2 \Gamma_{B}^{R} T_{R}} \\
& \propto \frac{\Delta}{T_{a}}\left(1-x \frac{\Delta}{T_{a}}+x^{2} \frac{\Delta^{2}}{T_{a}^{2}}+\ldots\right),
\end{aligned}
$$

with $x=\left(\Gamma_{B}^{L}-\Gamma_{B}^{R}\right) /\left(\Gamma_{B}^{L}+\Gamma_{B}^{R}\right)$. This demonstrates that even $\Delta$ terms, i.e. thermal rectification, are directly linked to the parametric asymmetry. Fig. 2 further displays the tunability of a spin-subsystem-metal junction. In the classical limit $\left(\omega<T_{\nu}\right)$ rectification can be substantial, while in the quantum regime the effect is suppressed. Modifying the system-metal coupling strength largely controls the rectification ratio (inset).

To summarize, while previous studies were focused on a specific realization, typically limited to the classical regime, based on numerical simulations, we have analytically deduced sufficient condition for the onset of thermal rectification in generic hybrid structures at the level of the quantum master equation: (i) The reservoirs should be made dissimilar, e.g. rectification emerges in an anharmonic junction where the $L$ and $R$ segments have different potentials. (ii) The contacts could be of the same type, but their statistics should differ from that of the system, combined with some parametric asymmetry, e.g. a boson-spin-boson junction rectifies heat when $\Gamma_{B}^{L} \neq \Gamma_{B}^{R}$. Our study applies to various interfaces: metals, insulators and noninteracting spins. The central unit could represent a radiation mode, a vibrational mode, or an electronic excitation.

Anharmonic interactions were in particular pointed out responsible for thermal rectification. While it is obvious that perfectly harmonic systems cannot rectify heat [4], not all anharmonic-asymmetric systems do bring in the effect. Consider for example a three-segment nonlinear oscillators chain where all units have identical potentials, but the central part is asymmetrically connected to the two terminals. According to our analysis this system will not rectify heat.

Our study manifests control over energy transfer at the nanoscale, important for cooling electronic and mechanical devices and for controlling molecular reactivity. We also demonstrated that thermal rectification is an abundant effect that could be observed in a variety of systems, phononic [17], electronic [18], and photonic [6, 22].

Acknowledgement This work was supported by the University of Toronto Start-up Funds.
[1] V. P. Carey et al., Nanoscale and Microscale Thermophysical Engineering 12, 1 (2008).

[2] A. Nitzan and M. A. Ratner, Science, 300, 1384 (2003).

[3] Z. Wang et al. Science 317, 787 (2007).

[4] F. Bonetto, J. Lebowitz, and L. Rey-Bellet, Mathematical Physics 2000 (World Scientific, Singapore, 2000), pp. 128150.

[5] C. W. Chang et al., Phys. Rev. Lett. 101, 075903 (2008).

[6] M. Meschke, W. Guichard, and J. P. Pekola, Science 444, 187 (2006).

[7] J. M. Taylor, C. M. Marcus, and M. D. Lukin, Phys. Rev. Lett. 90, 206803 (2003).

[8] E. H. G. Backus et al., Science 310, 1790 (2005).
[9] F. Giazotto, et al. Rev. Mod. Phys. 78, 217 (2006).

[10] M. Terraneo, M. Peyrard, and G. Casati, Phys. Rev. Lett. 88, 094302 (2002).

[11] B. Li, L. Wang, and. G. Casati, Phys. Rev. Lett. 93, 184301 (2004).

[12] D. Segal and A. Nitzan, Phys. Rev. Lett. 94, 034301 (2005); J. Chem. Phys. 122, 194704 (2005).

[13] B. Hu, L. Yang, and Y. Zhang, Phys. Rev. Lett. 97, 124302 (2006).

[14] G. Casati, C. Mejia-Monasterio, and T. Prosen, Phys. Rev. Lett. 98, 104302 (2007).

[15] N. Yang, N. Li, L. Wang, and B. Li, Phys. Rev. B 76, 020301 (2007). 
[16] N. Zeng and J.-S. Wang, Phys. Rev. B 78, 024305 (2008).

[17] C. W. Chang et al., Science 314, 1121 (2006).

[18] R. Scheibner et al., New. J. Phys. 10 , 083016 (2008).

[19] L.-A. Wu and D. Segal, arXiv:0804.3371 1.

[20] All even $\Delta$ terms of $J$ have a contribution from the preceding odd term times $\operatorname{Tr}\left[\left(H_{L}^{0}-H_{R}^{0}\right) \rho_{T}\right]$. The following discussion thus holds for higher orders as well.
[21] H.-P. Breuer and F. Petruccione, The Theory of Open Quantum Systems, Oxford University Press, New York, New York, (2002),

[22] D. Segal, Phys. Rev. Lett. 100, 105901 (2008).

[23] D. Segal, Phys. Rev. B 73, 205415 (2006). 